\title{
An Effective Tone Mapping Operator for High Dynamic Range Images
}

\author{
B. Kh. Barladian, A. G. Voloboi, V. A. Galaktionov, and E. A. Kopylov \\ Keldysh Institute of Applied Mathematics, Russian Academy of Sciences, \\ Miusskaya pl. 4, Moscow, 125047 Russia \\ e-mail: obb@gin.keldysh.ru \\ Received April 23, 2004
}

\begin{abstract}
Tone mapping operators are used to compress a large range of pixel luminances into a smaller range, which can be displayed on a monitor screen. In this paper, an effective and easy-to-use tone mapping operator based on the latest ideas developed in this field is presented. The parameter estimation process relies on the sampling method. Special attention is given to the robustness of the algorithm for the parameter estimation. The suggested tone mapping operator ensures good quality of images and almost does not require manual parameter tuning.
\end{abstract}

\section{INTRODUCTION}

High dynamic range images are now commonly used in modern computer graphics. Initially, such images were produced mainly by physically based lighting simulation systems. Today, high dynamic range maps of real scenes can even be produced by pretty standard cameras. It is only required to have a few differently exposed photographs of the scene [1], or a panoramic video scan of it $[2,3]$. It seems likely that, in the nearest future, photo and video cameras will directly capture high dynamic range images [4-6].

Images with high dynamic range of brightness have many advantages compared to ordinary, low dynamic range, images [7]. The use of such images is very useful in a number of applications $[8,9]$. However, the use of these images results in many difficulties associated with displaying them on standard devices (graphic monitors, printers, etc.), the dynamic range of which is considerably smaller than the dynamic range of real scenes. To represent high dynamic range images on standard display devices, we need to compress real data to fit the displayable range of these devices. This compression is referred to as tone mapping, or tone reproduction. The quality of such operators is estimated by the level of details preserved in the image, local contrast, and absence of artifacts. The operation rate of the algorithm and the amount of memory required are also very important characteristics, since, in a number of cases, there is a need in the real-time mode of the algorithm operation.

The practical need for such algorithms stimulated the emergence of a large number of works devoted to the development of efficient tone operators for images with high dynamic range of brightness. A number of papers were presented on the SIGGRAPH, Eurographics, and Graphicon conferences [9-15]. Most of the algorithms presented in these works can successfully be applied to both real images (for example, photographs) with high dynamic range of brightness and to the images created by physically based lighting simulation system. Actually, the use of the tone mapping operator in simulation systems has its own specific features related to the anti-aliasing technology, which will be discussed below.

The tone mapping operators can be classified into two large groups: (1) global (spatially invariant) and (2) local (spatially variant) operators. [1, 11, 16-18].

Global operators compress the luminance of each pixel using a fixed curve, which is constructed on the basis of some averaged image characteristics. A log average luminance level is often used as one of such image characteristic. The advantage of these algorithms is their simplicity; however, image details are often lost in light or dark areas of images, where the dynamic range is especially large.

In local operators, the transformation of the luminance in each pixel depends on the average luminance level in a local neighborhood of the transformed pixel. For the algorithms of this kind, the most difficult and computationally expensive problem is to correctly determine the size of the local neighborhood for each pixel. If this size is determined incorrectly, various artifacts may arise (example of such artifacts are presented in [12]).

The use of local operators for transforming images created in physically correct simulation systems involves certain specific difficulties in addition to the above-mentioned problems related to the artifacts and large amount of computations. The obtaining of highquality images requires special anti-aliasing of image contours. The algorithms used for these purposes, as rule, divide the screen pixel into smaller components, 
which increases the size of the image obtained by more than an order of magnitude. Then, the final high-quality image is obtained by averaging the subpixels. The tone mapping operator should be used on the subpixel level. Otherwise, the quality of the resulting image can decrease considerably. The use of local operators for images of such great size results in additional difficulties, since this requires much memory and considerably increases the processing time. The requirements on the memory size become especially critical when the set of images is created for the purpose of animation. In this case, the tone mapping operator must also provide smooth variation of the image brightness from one frame to another.

Taking into account all above-mentioned difficulties, we have developed a tone mapping operator and a robust algorithm for estimating its parameters.

\section{ALGORITHM}

Our tone mapping operator is based on the revised Tumblin-Rushmeier tone reproduction operator [18] and an additional formula suggested by Reinhard et al., $[12,13]$, which compresses mainly high luminances.

The tone mapping operator suggested by Tumblin and Rushmeier [19] uses a model of brightness preservation, which is based on a mathematical model of human vision [20]. The goal of this model is to keep fixed the ratio of the brightness of a scene perceived on the display to its real counterpart for any lighting condition. Our operator is based on the revised TumblinRushmeier operator, which reduces displayed contrasts for very dark scenes, preventing thus contrast reversals and exaggerations.

The revised tone reproduction operator is defined as $([17,18])$

$$
L_{d}=m\left(L_{w a}\right) L_{d a}\left(\frac{L_{w}}{L_{w a}}\right)^{\left(\frac{\gamma_{w}}{\gamma_{d}}\right)},
$$

where $L_{d a}$ is the display adaptation luminance (typically, between 10 and $\left.30 \mathrm{~cd} / \mathrm{m}^{2}\right) ; L_{w a}$ is the scene adaptation luminance determined in terms of the scene luminances (physical pixel luminances $L_{w}$ ) as

$$
\begin{gathered}
\log \left(L_{w a}\right) \\
=\operatorname{mean}\left\{\log \left(L_{w}+2.3 \times 10^{-5} \mathrm{~cd} / \mathrm{m}^{2}\right)\right\} ;
\end{gathered}
$$

$\gamma_{d}$ and $\gamma_{w}$ are determined from Stevens' contrast sensitivity formula

$$
\gamma\left(L_{a}\right)=\left\{\begin{array}{l}
2.655, \text { if } L_{a}>100 \mathrm{~cd} / \mathrm{m}^{2} \\
1.855+0.4 \log _{10}\left(L_{a}\right. \\
\left.+2.3 \times 10^{-5}\right), \text { otherwise; }
\end{array}\right.
$$

(assuming that the human eye is adapted to the display, we may set $\gamma_{d}=\gamma\left(L_{d a}\right)$ and $\left.\gamma_{w}=\gamma\left(L_{w a}\right)\right)$; and $m\left(L_{w a}\right)$ is a coefficient depending on the scene adaptation luminance, which prevents anomalously gray night images,

$$
m\left(L_{w a}\right)=\left(\sqrt{C_{\max }}\right)^{\left(\gamma_{w d}-1\right)} .
$$

Here, $C_{\max }$ is the maximum possible display contrast (typically, from 30 to 100), and

$$
\gamma_{w d}=\left(\frac{\gamma_{w}}{1.855+0.4 \log \left(L_{d a}\right)}\right)
$$

The multiplier $m$ increases monotonically the display brightness as the scene adaptation luminance $L_{w a}$ grows up to the upper vision limits.

We applied these formulas to the luminances computed from the RGB triples using the formula

$$
\begin{gathered}
L_{w}(x, y)=0.252 R(x, y) \\
+0.664 G(x, y)+0.084 B(x, y) .
\end{gathered}
$$

To avoid excessive computations and the use of great amount of memory when calculating the scene adaptation luminance, we used sampling of pixels from a relatively rare grid, so that only a small part of pixels (typically, about $1 \%$ ) was used for these calculations. Some details of these computations and the measures used for ensuring robustness of the parameter estimation are discussed in the next section.

In the general case, the use of the revised TumblinRushmeier operator may result in that the luminance of some pixels on the display corresponding to the brightest part of the scene will exceed the maximum allowable monitor brightness of the displayable range. To overcome this difficulty, our operator uses the following formula, introduced by Reinhard et al. [12], for compressing high luminances:

$$
L_{d f}(x, y)=\frac{L_{d}(x, y)\left(1+\frac{L_{d}(x, y)}{L_{\mathrm{white}}^{2}}\right)}{1+L_{d}(x, y)},
$$

where $L_{\text {white }}$ is the smallest luminance mapped onto the pure white. For images with very high dynamic range, $L_{\text {white }}$ may be set equal to any sufficiently large value (typically, close to the maximum scene luminance). For images with low and medium dynamic ranges, the correct selection of this parameter is of critical importance.

The selection of this parameter is discussed in detail in the next section.

\section{PARAMETER ESTIMATION}

In the previous section, it has been shown that the suggested tone mapping operator depends on the following two parameters: the scene adaptation luminance $L_{w a}$ and the white point $L_{\text {white }}$. The sampling from the rare grid is used for the estimation of both these parameters. The estimation is divided into two stages.

First, the scene adaptation luminance is estimated by means of Eq. (2), and the white point is selected such 

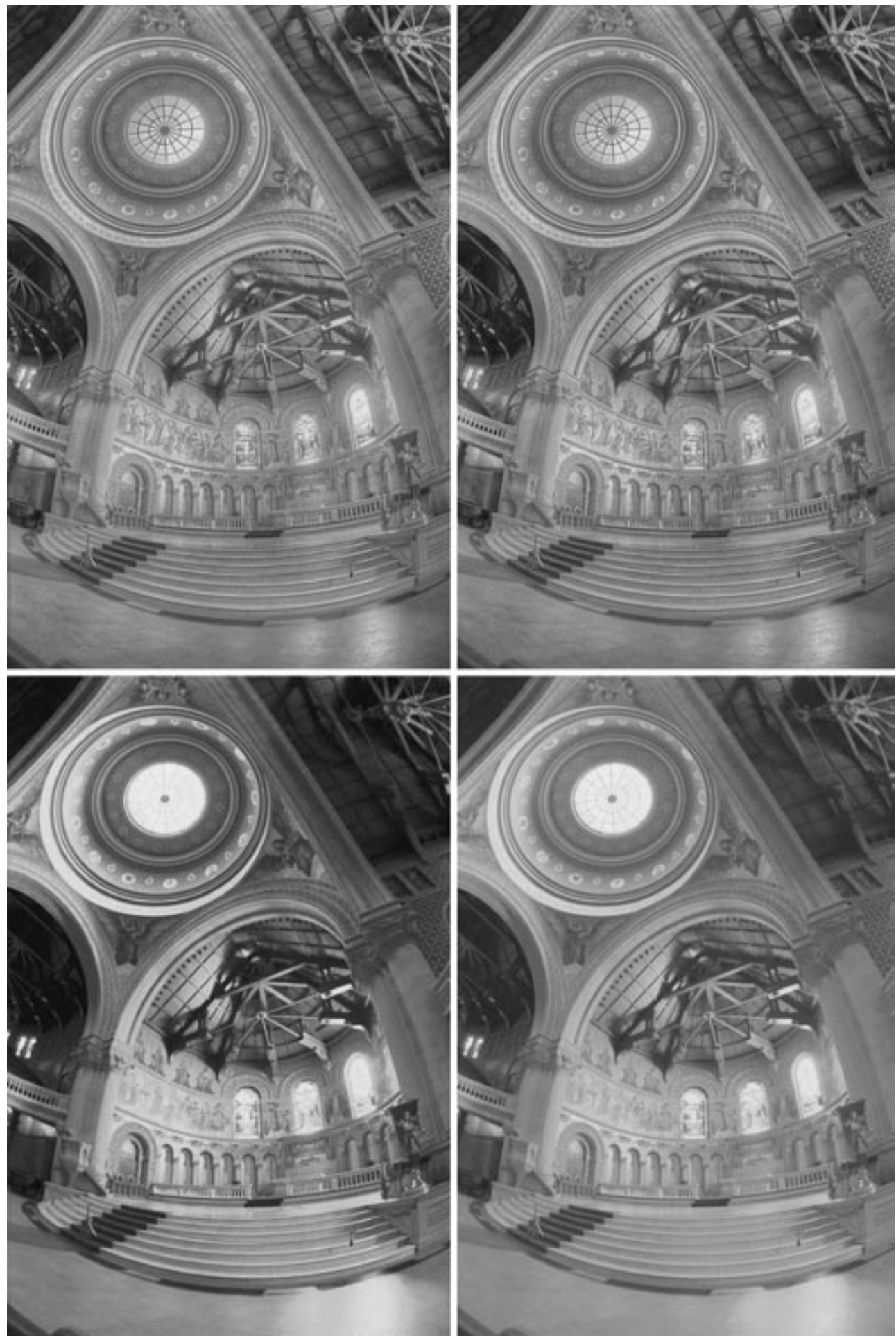

Fig. 1. Stanford Memorial Church. The left top image is obtained by the Gradient Domain method [10]; the right top image, by the LCIS method [16]; the left bottom image, by the method from [22]; and the right bottom image, by our method.

that the luminance of approximately $1 \%$ of all scene pixels exceeds this value.

This approach works satisfactory for most scenes; however, it yields poor results for scenes with large dark areas: the most interesting part of the image is oversaturated. The constant $2.3 \times 10^{-5} \mathrm{~cd} / \mathrm{m}^{2}$ suggested in [18] allows us to avoid zero values under the logarithm sign; however, it is too small for providing reasonable scene adaptation luminance values for these scenes. To overcome this difficulty, we exclude the 

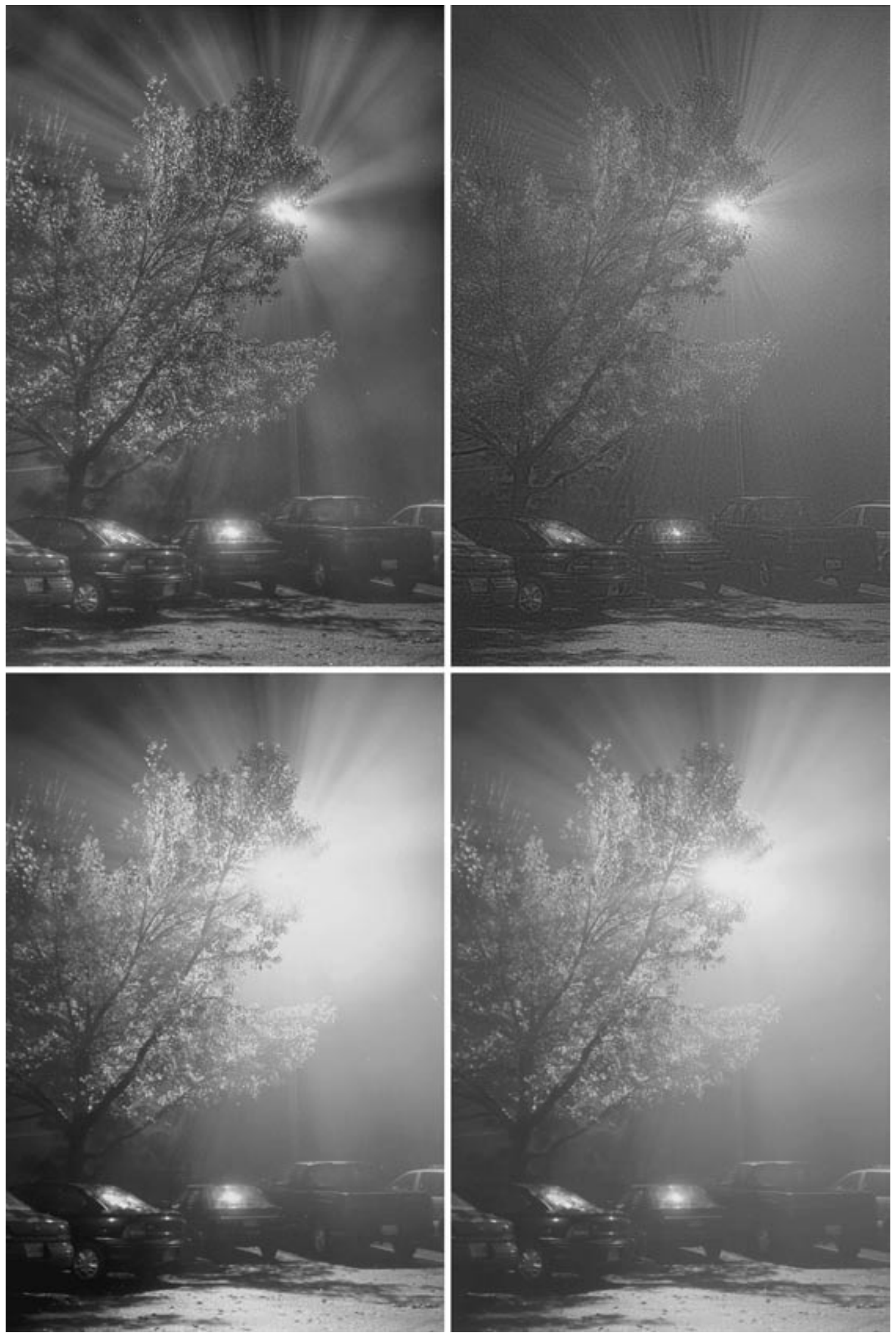

Fig. 2. Streetlight on a Foggy Night. The left top image is obtained by the Gradient Domain method [10]; the right top image, by the LCIS method [16]; the left bottom image, by the method from [22]; and the right bottom image, by our method. 

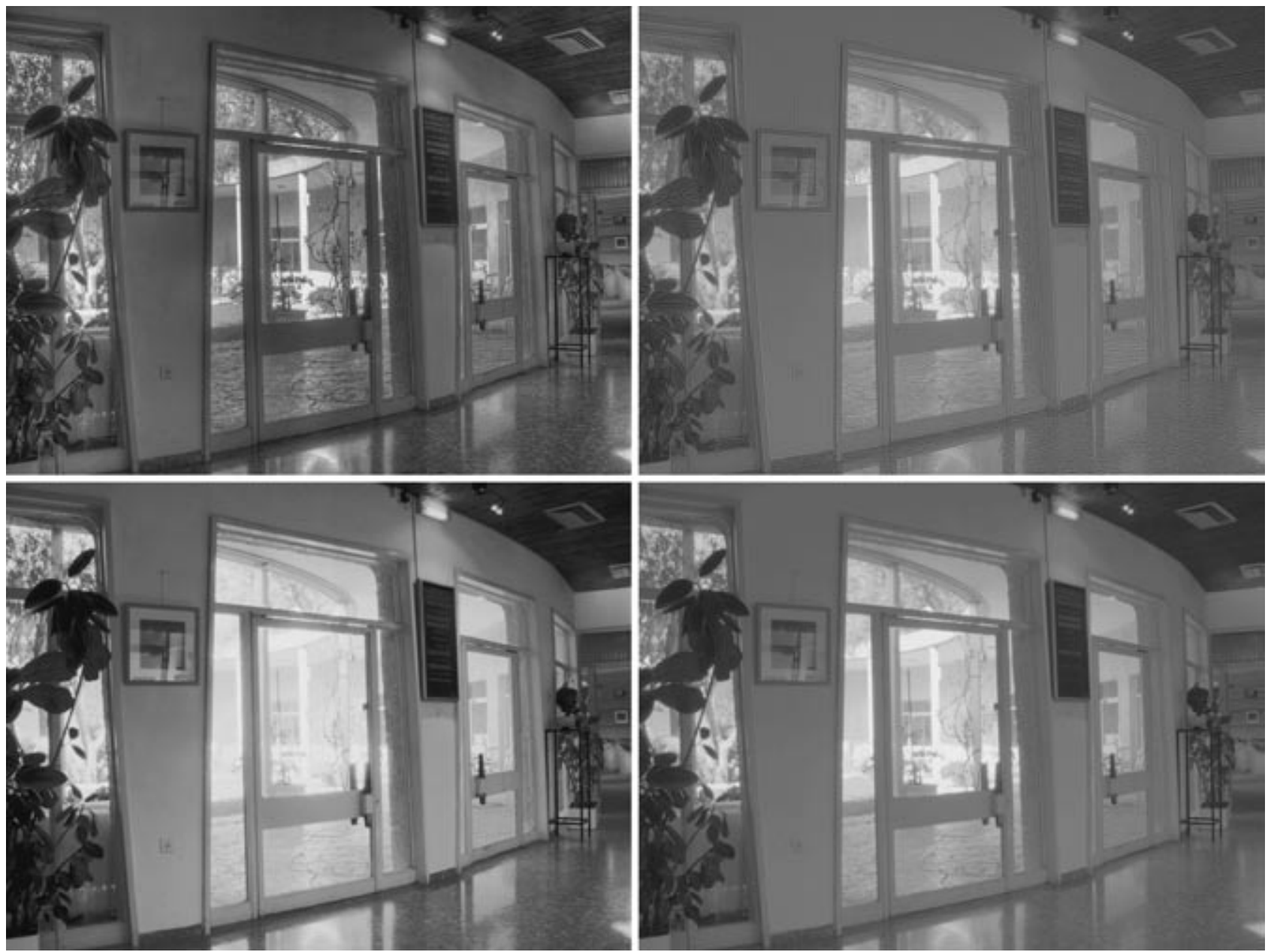

Fig. 3. Belgium House. The left top image is obtained by the Gradient Domain method [10]; the right top image, by the LCIS method [16]; the left bottom image, by the method from [22]; and the right bottom image, by our method.

darkest pixels from the calculations when determining the scene adaptation luminance by formula (2). The following heuristic formula was introduced for determining the threshold level:

$$
L_{\text {trsh }}=\min \left(L_{w a} / 20, L_{\text {white }} / 100\right) .
$$

Another improvement concerns the fine-tuning of the white point. The value selected at the first stage ensures nice results when the white level is exceeded in a small number of pixels, and, thus, the oversaturation at these points has little effect on the image perception (because brightness of the major part of the image is considerably lower than the selected white level). However, this approach does not work properly when many image pixels have the luminance value close to this white point level. In such a case, this part of the image becomes oversaturated. To overcome this difficulty, the white point level is selected such that the initially selected white point luminance corresponds strictly to the given screen luminance. The empiric value 0.98 (for the canonical range $[0,1]$ ) yields nice results for the majority of the scenes tested. We denote this display white threshold by $L_{d w t}$. The new white point level is found by solving jointly equations derived from (1)-(6), with regard to the already calculated quantities $L_{w a}$ and $L_{d w t}$ and the initial value of $L_{\text {white }}$, in the space of physical luminance values:

$$
\left\{\begin{array}{c}
L_{d}=m\left(L_{w a}\right) L_{d a}\left(\frac{L_{\mathrm{white}}}{L_{w a}}\right)^{\left(\frac{\gamma_{w}}{\gamma_{d}}\right)} \\
L_{d w t}=\frac{L_{d}\left(1+\frac{L_{d}}{L_{\mathrm{white}}^{2}}\right)}{1+L_{d}}
\end{array}\right.
$$

\section{RESULTS AND DISCUSSION}

Our method has been implemented in several products of INTEGRA Inc. [21]. It works fine for a representative set of high dynamic range images generated in these systems. We also have tested our method on various high dynamic range images of real scenes. In all 

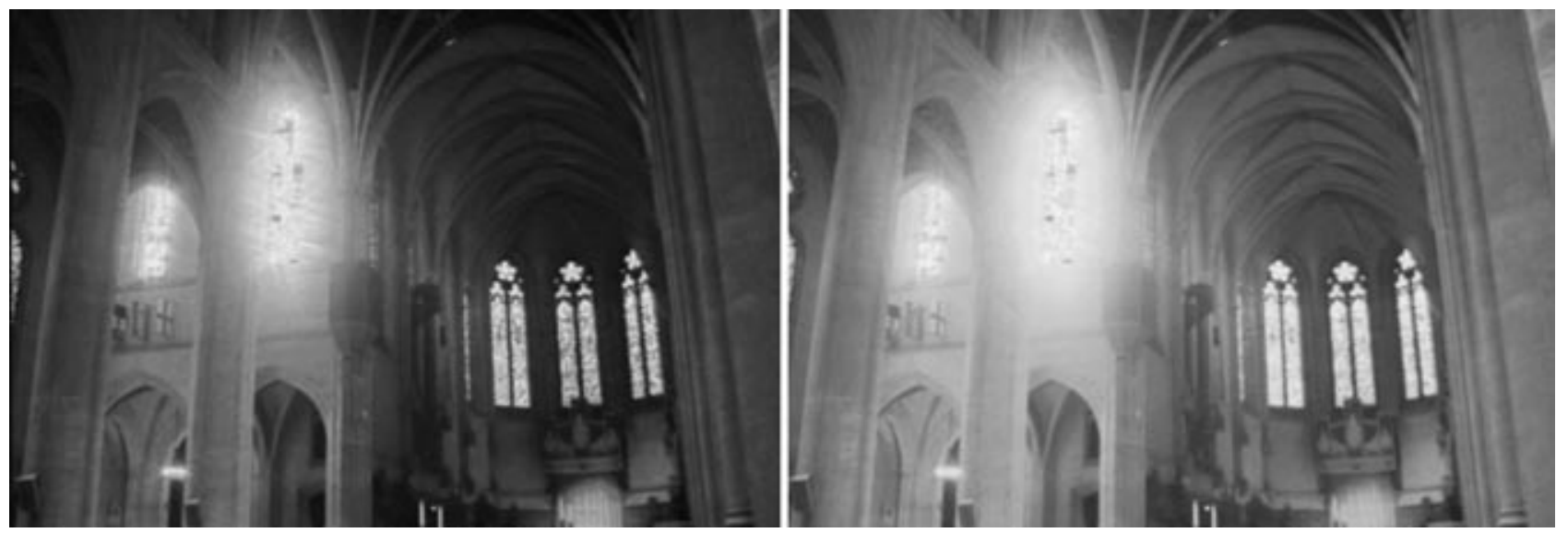

Fig. 4. Nave. The left image is obtained by Reinhard [12], and the right image, by our method.

cases, our method produced satisfactory results without additional parameter tuning. Results of some of these experiments are presented in the figures below. ${ }^{1}$

Figure 1 shows four different renderings of the "Stanford Memorial Church" picture [7, 10]. The dynamic range for this picture exceeds $250000: 1$. The left top image was produced by the Fattall method [10], the right top image was obtained by means of Tumblin and Turk's LCIS method [16], the left bottom image, by using the method suggested in [22], and the right bottom image, by our method.

Figures 2 and 3 present a similar comparison for the "Streetlight on a Foggy Night" and "Belgium House" pictures [10]. The dynamic ranges for these images exceed $100000: 1$ and $500000: 1$, respectively.

Figure 4 compares two images of the "Nave" picture [7]. The left image was produced by the Reinhard method [12], and the right one, by ours. As can be seen, our method is capable of displaying fine details in both the bright and dark parts of the picture. A disadvantage of the method consists in the reduction of the contrast, which is especially noticeable in bright parts of the image. Our method is free of artifacts typical of the LCIS method. For example, the floor in the image in Fig. 1 obtained by the LCIS method seems disagreeably rough, while the image in Fig. 2 is too grainy.

Note also that, among the methods under comparison, ours is the most efficient. For example, the processing of an image of 3600 by 2060 pixels by the Reinhard method [12] took about 5 minutes, whereas our method coped with this problem for 20 seconds on the same computer. Thus, the performance of our method is 15 times greater than that of the Reinhard method.

The least loss of image details under the condition of preserving the contrast and without artifacts was achieved by the Domain Gradient method [10]. This method is also considered to be the fastest one among

\footnotetext{
${ }^{1}$ Full-color images can be found at http://www.keldysh.ru/pages/ cgraph/articles/index.html
}

the known local methods. Still, the processing time in this method is considerable and varies from $1.1 \mathrm{~s}$ for an image of 512 by 384 pixels to $5 \mathrm{~s}$ for an image 1130 by 751 pixels on a Pentium 4, $1800 \mathrm{MHz}$ [10].

The processing of such images by our method takes from $0.26 \mathrm{~s}$ for an image 768 by 512 pixels to $0.56 \mathrm{~s}$ for a 1025-by-769-pixel image on a slower Pentium 3866 MHz.

\section{ACKNOWLEDGMENTS}

This work was supported by the Russian Foundation for Basic Research, project no. 04-01-00520, and by INTEGRA Inc. (Tokyo, Japan).

\section{REFERENCES}

1. Dicarlo, J., and Wandell, B., Rendering High Dynamic Range Images, Proc. of the SPIE: Image Sensors 3965, 2000, pp. 392-401.

2. Aggarwal, M. and Ahuja, N., High Dynamic Range Panoramic Imaging, Proc. IEEE ICCV, 2001, vol. I, pp. 2-9.

3. Schechner, Y.Y. and Nayar, S.K., Generalized Mosaicing, Proc. IEEE ICCV, 2001, vol. I, pp. 17-24.

4. Aggarwal, M. and Ahuja, N., Split Aperture Imaging for High Dynamic Range, Proc. IEEE ICCV, vol. II, pp. 10-17.

5. Nayar, S.K. and Mitsunaga, T., High Dynamic Range Imaging: Spatially Varying Pixel Exposures, Proc. IEEE CVPR, 2000.

6. SpheronVR, http: //www.spheron.com/

7. Debevec, P.E. and Malik, J., Recovering High Dynamic Range Radiance Maps from Photographs, Annual Conference Series: ACM SIGGRAPH (Proc. of the SIGGRAPH 97 Conf., 1997), Whitted, T., Ed., AddisonWesley, 1997, pp. 369-378.

8. Durand, F. and Dorsey, J., Interactive Tone Mapping, Eurographics Workshop on Rendering, 2000, pp. 219-230.

9. Durand, F. and Dorsey, J., Fast Bilateral Filtering for the Display of High-Dynamic-Range Images, ACM Trans. Graphics, 2002, vol. 21, no. 3 (Proc. of SIGGRAPH 2002). 
10. Fattall, R., Lischinski, D., and Werman, M., Gradient Domain High Dynamic Range Compression, ACM Trans. Graphics, 2002, vol. 21, no. 3 (Proc. of SIGGRAPH 2002).

11. Ferwerda, J., Fundamentals of Spatial Vision, in Applications of Visual Perception in Computer graphics. Siggraph'98 Course Notes, 1998.

12. Reinhard, E., Stark, M., Shirley, P., and Ferwerda, J., Photographic Tone Reproduction for Digital Images, ACM Trans. Graphics, 2002, vol. 21, no. 3 (Proc. of SIGGRAPH 2002).

13. Reinhard, E., Parameter Estimation for Photographic Tone Reproduction, J. Graphics Tools, 2002, vol. 7, no. 1, pp. 45-52.

14. Barladian, B., Robust Parameter Estimation for Tone Mapping Operator, Proc. of Graphicon'2003, Moscow, 2003.

15. Kang, S.B., Uyttendaele, M., Winder, S., and Szelliski, R., High Dynamic Range Video, Proc. of SIGGRAPH 2003, 2003.

16. Tumblin, J. and Turk, G., LCIS: A Boundary Hierarchy for Detail-Preserving Contrast Reduction, Annual Con- ference Series: Computer Graphics Proceedings (Siggraph 1999), Rockwood, A., Ed., Los Angeles: AddisonWesley, 1999, pp. 83-90.

17. Tumblin, J., Hodgins, J.K., and Guenter, B.K., Two Methods for Display of High Contrast Images, ACM Trans. Graphics, 1999, vol. 18, no. 1, pp. 56-94.

18. Tumblin, J., Three Methods For Detail-Preserving Contrast Reduction for Displayed Images, PhD Dissertation, Georgia Inst. of Technology, 1999.

19. Tumblin, J. and Rushmeier, H., Tone Reproduction for Computer Generated Images, IEEE Comput. Graphics Appl., 1993, vol. 13, no. 6, pp. 42-48.

20. Stevens, S.S. and Stevens, J.C., Brightness Function: Parametric Effects of Adaptation and Contrast, J. Opt. Soc. Am., 1960, vol. 50, no. 11, p. 1139A.

21. http://www.integra.jp

22. Ward Larson, G., Rushmeier, H., and Piatko, C., A Visibility Matching Tone Reproduction Operator for High Dynamic Range Scenes, IEEE Trans. Visualization Comput. Graphics, 1997, vol. 3, no. 4, pp. 291-306. 